\title{
单元轨道线性组合近似理论 理论和数值计算方法
}

叶元杰杨忠志唐敖决

(吉林大学理论化学研究所, 长春)

迄今为止, 人们对于具有重复单元的分子体系的量子化学计算大多采用 Born-Kármen 周 期性边界条件进行近似 ${ }^{[1-3]}$. 但是实际存在的分子体系并不满足周期性边界条件. 已有许多化 学家采用非周期边界条件处理共轭分子体系 ${ }^{[4-9]}$, 得到了许多很有意义的成果, 然而所采用的

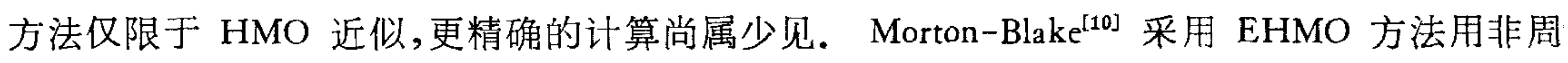
期性边界条件计算了一些分子体系, 对此进行了探讨, 但所用计算方法不能用于较大的体系。

本文作者曾提出单元轨道线性组合近似理论 ${ }^{[11}$ 并得到了特殊情况下的解析解. 本文在此 基础上提出了完整的近似方法, 对于线型分子, 所得结果为求解分块三对角矩阵的广义本征值 问题.

对矩阵本征值问题进行数值求解的计算技术已有很大的发展 ${ }^{[12-18]}$. 近年来, 人们在研究 无序体系的过程中发展了许多求解类似问题的计算方法 ${ }^{[14]}$. 其中, Dean ${ }^{[12,13]}$ 等人为了计算 无序体系的振动问题证明了负本征值定理, 可以精确计算本征值. 吴式玉 ${ }^{[14,15]}$ 等人采用 Green 函数法在此基础上计算三对角矩阵的本征向量. 在以上作者工作的基础上，本文证明了求解 实对称块状三对角矩阵广义本征值问题的负本征值定理, 推导了直接计算本征向量的方法.

设分子体系可以分为 $N$ 个相对独立的单元, 这些单元可以相同, 如在聚合物中, 也可以不 同, 如在生物大分子内, 每个单元均已完成了量子化学计算, 得到了 $m_{n}$ 个单元轨道, 则整个体 系的分子轨道可以写为所有单元轨道的线性组合:

$$
\psi=\sum_{n=1}^{N} \sum_{j=1}^{m_{n}} C_{j}(n) \Phi_{j}(n),
$$

假设仅单元内和相邻单元之间的共振积分和重迭积分不为零, 则对于线型分子, 由线性变分法 可得如下广义本征方程:

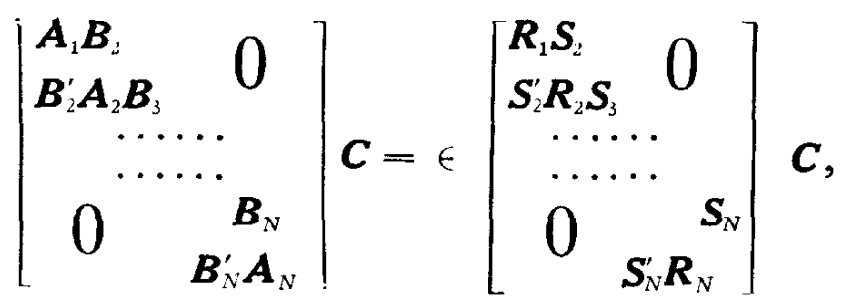

其中, $\boldsymbol{A}_{n}, \boldsymbol{R}_{n}$ 为 $m_{n} \times m_{n}$ 方阵, $\boldsymbol{B}_{n}$ 与 $\boldsymbol{S}_{n}$ 可以不是方阵. 方程(2)可以简记为

$$
H C=\in S C \text {. }
$$

本文 1985 年 1 月 30 日收到. 
当体系由重复单元构成时,再进一步假设共振积分和重迭积分与单元序数无关, 即为文献 [11] 所得的方程 (2).

引进实参量 $\chi$, 设 $\chi$ 不等于方程 (3) 的本征值, 作矩阵 $\boldsymbol{H}-\chi \boldsymbol{S}$, 并将其分解为一个下三. 角矩阵与一个上三角分块矩阵的乘积:

$$
\boldsymbol{H}-\chi \boldsymbol{S}=\boldsymbol{L}(\chi) \cdot \boldsymbol{U}(\chi)
$$

其中

$$
\boldsymbol{L}(\chi)=\left[\begin{array}{ccc}
\boldsymbol{I}_{m_{1}} & & 0 \\
\boldsymbol{L}_{2} \boldsymbol{I}_{m_{2}} & & \\
\ddots & \ddots & \\
\ddots & & \\
0 & \boldsymbol{L}_{N} \boldsymbol{I}_{m_{V}}
\end{array}\right], \boldsymbol{U}(\chi)=\left[\begin{array}{ccc}
\boldsymbol{U}_{1} \boldsymbol{V}_{1} & 0 \\
\boldsymbol{U}_{2} \boldsymbol{V}_{2} & \\
\ddots & \ddots & \\
0 & \boldsymbol{U}_{N-1} \boldsymbol{V}_{N-1} \\
& & \boldsymbol{U}_{N}
\end{array}\right]
$$

$\boldsymbol{L}(\chi) 、 \boldsymbol{U}(\chi)$ 的分块方法与 $\boldsymbol{H} 、 \boldsymbol{S}$ 相同. 由矩阵分块乘法可得如下递推关系:

$$
\begin{aligned}
& \boldsymbol{U}_{1}=\boldsymbol{A}_{1}-\chi \boldsymbol{R}_{1}, \\
& \boldsymbol{U}_{n}=\boldsymbol{A}_{n}-\chi \boldsymbol{R}_{n}-\left(\boldsymbol{B}_{n}^{\prime}-\chi \boldsymbol{S}_{n}^{\prime}\right) \boldsymbol{U}_{n-1}^{-1}\left(\boldsymbol{B}_{n}-\chi \boldsymbol{S}_{n}\right) .
\end{aligned}
$$

假设方程 (3) 的本征值非简并, 则用与文献 [12]类似的方法可以证明广义本征方程 (3) 的负本 征值定理如下:

定理 1 方程 (3) 在区间 $-\infty<\chi^{\prime} \leqslant \chi$ 中的本征值的个数等于矩阵 $\boldsymbol{U}_{n}(\chi)(n=1,2$, $\cdots, N)$ 的负本征值的总个数.

证由(4)式和(5)式可知

$$
\operatorname{det}(\boldsymbol{H}-\chi \boldsymbol{S})=\prod_{n=1}^{N} \operatorname{det} \boldsymbol{U}_{n}(\chi),
$$

由于 $\chi$ 不是方程 (3) 的本征值, 所以 $\operatorname{det}(\boldsymbol{H}-\chi \boldsymbol{S}) \neq 0$, 而知 $\operatorname{det} \boldsymbol{U}_{n}(\chi)$ 均非零, 从而

$$
\operatorname{det} \boldsymbol{U}_{N}=\frac{\operatorname{det}(\boldsymbol{H}-\chi \boldsymbol{S})}{\prod_{n=1}^{N-1} \operatorname{det} \boldsymbol{U}_{n}},
$$

于是知 $\operatorname{det} \boldsymbol{U}_{N}$ 的零点必为 $\operatorname{det}(\boldsymbol{H}-\chi \boldsymbol{S})$ 的零点.

对于充分大的 $\chi>0$, 由(6)式可知 $\boldsymbol{A}_{n}, \boldsymbol{B}_{n}$ 相对于 $\chi \boldsymbol{R}_{n}$ 和 $\chi \boldsymbol{S}_{n}$ 均为小量,且可知 $\boldsymbol{U}_{1}$ 的本征值均为负值, $\boldsymbol{U}_{1}^{-1} \sim-\frac{1}{x} \boldsymbol{R}_{1}^{-1}$. 显然 $\boldsymbol{R}_{1}^{-1}$ 亦为对称正定矩阵. 又由 (6) 的第二式知

$$
\boldsymbol{U}_{2} \sim-\chi \boldsymbol{R}_{2}-\chi \boldsymbol{S}_{2}^{\prime} \boldsymbol{R}_{1}^{-1} \boldsymbol{S}_{2}=-\chi \boldsymbol{T}_{2},
$$

从 $\boldsymbol{R}_{1}^{-1}$ 的对称正定性质知有非异矩阵 $Q$ 使 $\boldsymbol{R}_{\mathbf{1}}^{-1}=Q^{\prime} Q$, 而有

$$
\left|\boldsymbol{S}_{2}^{\prime} \boldsymbol{R}_{1}^{-1} \boldsymbol{S}_{2}\right|=\left|\boldsymbol{S}_{2}^{\prime} Q^{\prime} Q \boldsymbol{S}_{2}\right|=\left|\boldsymbol{P}^{\prime} \boldsymbol{P}\right| \geqslant 0,
$$

由此即知 $\boldsymbol{U}_{2}$ 的本征值均小于零且 $\boldsymbol{T}_{2}$ 为对称正定矩阵. 依此类推, 可知此时诸 $\boldsymbol{U}_{n}$ 的本征值 均小于零. 同样可知, 当 $\chi \rightarrow-\infty$ 时, 诸 $\boldsymbol{U}_{n}$ 的本征值均大于零. 由(7)式可知诸 $\boldsymbol{U}_{n}$ 的本征 值的数目之和等于 $\operatorname{det}(\boldsymbol{H}-\chi \boldsymbol{S})$ 的零点个数. 从而当 $\chi$ 从一舟变化到充分大的 $\chi$ 时, 诸 $\boldsymbol{U}_{n}$ 的负本征值的总个数从 $O$ 变到 $M, M=\sum_{n=1}^{N} m_{n}$.

又设 $\boldsymbol{U}_{n}(n<N)$ 最小的本征值为 $u_{k}$, 本征向量为 $\boldsymbol{V}_{k}$, 当 $u_{k}$ 充分小时, $\frac{1}{u_{k}}$ 为大数, 而 
有

$$
\begin{aligned}
\boldsymbol{U}_{n}^{-1} & =\frac{1}{u_{k}} \boldsymbol{V}_{k} \boldsymbol{V}_{k}^{\prime}+\sum_{i \neq k} \frac{1}{u_{i}} \boldsymbol{V}_{i} \boldsymbol{V}_{\boldsymbol{i}} \\
& =\frac{1}{u_{k}} \boldsymbol{V}_{k} \boldsymbol{V}_{k}^{\prime}+O\left(\frac{1}{u_{k}}\right),
\end{aligned}
$$

于是

$$
\boldsymbol{U}_{n+1}=-\frac{1}{u_{k}}\left(\boldsymbol{B}_{n}^{\prime}-\chi \boldsymbol{S}^{\prime}\right) \boldsymbol{V}_{k} \boldsymbol{V}_{k}^{\prime}\left(\boldsymbol{B}_{n}-\chi \boldsymbol{S}_{n}\right)+o\left(\frac{1}{u_{k}}\right),
$$

亦即 $\boldsymbol{U}_{n+1}$ 有一个相当于 $-\frac{1}{u_{k}}$ 的充分大的本征值, 从而当 $\boldsymbol{U}_{n}$ 的一个本征值从负到正通过 零点变号时, 必有 $\boldsymbol{U}_{n+1}$ 的一个本征值从正到负通过无穷远点变号, 诸 $\boldsymbol{U}_{n}$ 的负本征值的总个 数保持不变. 由此可知能使诸 $\boldsymbol{U}_{n}$ 的负本征值的总个数发生变化的途径仅有:

i) $\boldsymbol{U}_{N}$ 的本征值通过零点变号;

ii) $U_{1}$ 的本征值通过无穷远点变号.

由物理意义知 $\boldsymbol{A}_{1}$ 与 $\boldsymbol{R}_{1}$ 均有界. 当 $\chi$ 有界时, $\boldsymbol{U}_{1}$ 无极点,因而途径 ii) 不可能. 由(8)式 可知, 仅当 $\chi$ 通过 $\operatorname{det}(H-\chi S)$ 的零点时, 诸 $\boldsymbol{U}_{n}$ 的负本征值的总个数发生变化, 增量为 \pm 1 . 由于 $\operatorname{det}(\boldsymbol{H}-\chi \boldsymbol{S})$ 的零点即为方程(3)的本征值, 综合以上讨论, 定理即已得证.

推论 方程 (3) 的非简并本征值为 $\left|\boldsymbol{U}_{N}(\chi)\right|$ 的零点.

证 由定理一的证明可知, 当 $\chi$ 通过 $|\boldsymbol{H}-\chi \boldsymbol{S}|$ 的零点时, $\boldsymbol{U}_{N}(\chi)$ 的最小本征值通 过零点变号. 于是得证.

定理 2 若 $\epsilon$ 为方程 (3) 的非简并本征值, $\boldsymbol{C}_{n}(n=1,2, \cdots, N)$ 为相应的本征向量 中属于第 $n$ 个单元的分量组成的列矩阵, 则 $\boldsymbol{C}_{n}$ 满足如下递推关系:

$$
\boldsymbol{C}_{n}=-\boldsymbol{U}_{n}^{-1}\left(\boldsymbol{B}_{n+1}-\in \boldsymbol{S}_{n+1}\right) \boldsymbol{C}_{n+1},
$$

且有

$$
\boldsymbol{U}_{N} \boldsymbol{C}_{N}=0 .
$$

证 将方程 (3)写为差分方程形式:

$$
\left(B_{n}^{\prime}-\epsilon \boldsymbol{S}_{n}^{\prime}\right) \boldsymbol{C}_{n-1}+\left(\boldsymbol{A}-\epsilon \boldsymbol{R}_{n}\right) \boldsymbol{C}_{n}+\left(\boldsymbol{B}_{n+1}-\epsilon \boldsymbol{S}_{n+1}\right) \boldsymbol{C}_{n+1}=0,
$$

边界条件为

$$
\boldsymbol{C}_{0}=\boldsymbol{C}_{N+1}=0
$$

可知

$$
\left(\boldsymbol{A}_{1}-\epsilon \boldsymbol{R}_{1}\right) \boldsymbol{C}_{1}+\left(\boldsymbol{B}_{2}-\epsilon \boldsymbol{S}_{2}\right) \boldsymbol{C}_{2}=0 .
$$

由推论知 $\left|\boldsymbol{U}_{n}(\epsilon)\right| \neq 0(n<N)$, 结合(6)式可得

$$
\boldsymbol{C}_{1}=-\boldsymbol{U}_{1}^{-1}\left(\boldsymbol{B}_{2}-\epsilon \boldsymbol{S}_{2}\right) \boldsymbol{C}_{2},
$$

反复代入(15)即可得递推关系(13)式.

当 $n=N$ 时,由(15)、(16)式可得

$$
\left(\boldsymbol{B}_{N}^{\prime}-\epsilon \boldsymbol{S}_{N}^{\prime}\right) \boldsymbol{C}_{N-1}+\left(\boldsymbol{A}_{N}-\epsilon \boldsymbol{R}_{N}\right) \boldsymbol{C}_{N}=0,
$$

用递推关系取代 $\boldsymbol{C}_{\mathrm{N}-1}$ 即得

$$
\left[\boldsymbol{A}_{N}-\epsilon \boldsymbol{R}_{N}-\left(\boldsymbol{B}_{N}^{\prime}-\epsilon \boldsymbol{S}_{N}^{\prime}\right) \boldsymbol{U}_{N-1}^{-1}\left(\boldsymbol{B}_{N}-\epsilon \boldsymbol{S}_{N}\right)\right] \boldsymbol{C}_{N}=0,
$$

结合 (6)式即知这恰为(14)式. 证毕. 
将定理 1 和定理 2 相结合, 即可以对方程(2)进行数值求解. 用定理 1 和对分区间法可计 算本征值,达到预定精度后，应用定理 2 计算相应的本征向量. 对极少数精度不足的本征向量 可用逆迭代技术 ${ }^{[16]}$ 改善. 我们取 $\boldsymbol{A}_{n}=\boldsymbol{A}_{0}, \boldsymbol{B}_{n}=\boldsymbol{B}_{0}, \boldsymbol{R}_{n}=\boldsymbol{R}_{0}, \boldsymbol{S}_{n}=\boldsymbol{S}_{0}, n=1, \cdots, N$, $m_{0}=4, N=50$ 作了算例，对分区间的最小宽度为 $10^{-9}$,采用 $\delta=\|(\boldsymbol{H}-\epsilon \boldsymbol{S}) \boldsymbol{C}\|$ 进行误差 分析,所得结果为 $\max \delta=0.199 \times 10^{-8}$. 由于此法仅对三对角块进行计算且逐个计算本征向 量, 易于实现内外存交换而节省内存. 采用双精度量, 对重复单元, 用本文方法占用主内存 $m^{2} \times(N+5) \times 8$ byte，对不重复单元为 $m^{2} \times N \times 5 \times 8$ byte，而 RSSM 法 ${ }^{[19]}$ 对两种情况 均占用 $m^{2} \times N^{2} \times 2 \times 8$ byte 的主内存.

\section{参考 文 献}

[1] André, J.-M., Brédas, J.-L., Delhalle, J., Ladik, J., Leroy, G. and Moser, G., Recent advances in the quantum theory of polymer, Lecture Notes in Physics, 113(1979).

[2] André, J.-M., Adv. Quan. Chem., 12(1980), 65.

[ 3 ] 严继民、张启元, 科学通报, 28(1983),9: 536.

[4] 磨敖庆、江元生、䁩国森、戴树珊，分子轨道图形理论，科学出版社，1980.

[5]张乾二、林连堂、王阅钦,休克尔矩阵图形方法,科学出凯社, 1981 .

[6]冯星洪、杨忠志、唐敖庆,科学通报，26(1981),1495.

[7] 李向平,化学学报, 40(1982)，699.

[8] 唐敖庆、李前树, 高等学校化学学报,3 (专刊) (1982), 125.

[9] 杨忠志、叶元杰、唐散大, 中国科学(待发表).

[10] Morton-Blake, D.A., Theorct, Chim, Acta(Berl), 51(1979), 85.

[11] 叶元杰、吾榕之、徐光宪,分子科学与化学研究, 4(1984)，3: 303.

[12] Dean, P. and Martin, J. L., Proc. Roy. Soc., A259(1960), 409.

[13] Dean, P., Rev. Mod. Plays., 44(1972),127.

[14] 吴式玉、郑兆勃,物理学进展, 1(1981),1：125;4(1984)，1：12.

[15] 郑兆勃,物理学报, $30(1981) ， i: 866$.

[16] 冯康,数值计算方法,国防工业出版社, 1978 .

[17] Wilkinson, J.H., The Algebraic Eigenvalue Problem, Clarendon Press, Oxford, 1965.

[18] Kågström, B. and Ruhe, A., Matrix pencils, Lecture Notes in Mathematics, 973(1983).

[19] 郭富印、冯国环、石中舌、朱耀祖，FORTRAN 算法汇编，第三分敷，国防工业出版社，1982，330-334。 